\title{
Determinação dos Valores Energéticos de Alguns Óleos e Gorduras para Pintos de Corte Machos e Fêmeas aos 21 Dias de Idade ${ }^{1}$
}

\section{Carla Cristina Cardoso Nascif ${ }^{2}$, Paulo Cezar Gomes ${ }^{3}$, Luiz Fernando Teixeira Albino ${ }^{3}$, Horacio Santiago Rostagno ${ }^{3}$}

\begin{abstract}
RESUMO - Foi realizado um ensaio biológico com pintos de corte no período de 21 a 32 dias de idade, com o objetivo de determinar os valores de energia metabolizável aparente (EMA) e energia metabolizável aparente corrigida pela retenção de nitrogênio (EMAn) do óleo de soja degomado, óleo de milho, óleo de canola, gordura de aves, gordura de coco, gordura suína, sebo bovino e de duas misturas, sendo a primeira composta de óleo de soja degomado + gordura de coco e a segunda de óleo de soja degomado + sebo bovino, ambas na proporção de 1:1. Foi realizado o método de coleta total de excretas no experimento. O período de duração do experimento foi de dez dias, sendo os cinco primeiros de adaptação e os cinco restantes de coleta de excretas. Foi observada diferença entre os tratamentos e entre os sexos, sendo os valores de EMAn obtidos para fêmeas, em média, 98\% dos valores de EMAn obtido para machos. Porém, não foi observada diferença entre a interação alimento x sexo. Os valores médios de EMAn encontrados para pintos de corte foram, respectivamente, para óleo de soja degomado, óleo de milho, óleo de canola, gordura de aves, gordura de coco, gordura suína, sebo bovino, para a mistura de óleo de soja degomado + gordura de coco e para a mistura de óleo de soja degomado + sebo bovino: 8.273, 8.582, 8.681, $8.511,7.487,7.536,7.227,8.029$ e $8.171 \mathrm{kcal} / \mathrm{kg}$ matéria natural
\end{abstract}

Palavras-chave: energia metabolizável, óleo, gordura, pintos de corte

\section{Determination of Energy Values of Some Oils and Fats for Broilers Chickens Males and Females in the 21 Day Old}

\begin{abstract}
One bioassay was carried out using 21-to32-day old broilers chicks to determine the values of apparent metabolizable energy (AME) and nitrogen-corrected apparent metabolizable energy (AMEn) of crude soybean oil, corn oil, canola oil, avian fat, coconut fat, swine lard, beef tallow, crude soybean oil + coconut fat and crude soybean oil + beef tallow, both latter in 1:1 proportion. The method of total excreta collection was used for the trials, which lasted 10 days each: the first five days for adaptation and the five remaining days for excreta collection. In the assay, difference was observed among the treatments and between the sexes, with the AMEn values obtained for the females accounting for $98 \%$ of the AMEn values obtained for the males, on average. The interaction feed $\mathrm{x}$ sex showed no difference. The mean values of AMEn observed for broilers chicks for crude soybean oil, corn oil, canola oil, avian fat, coconut fat, swine lard, beef tallow, crude soybean oil + coconut fat and crude soybean oil + beef tallow, $1: 1$, were, respectively: 8,273, 8,582, 8,681, $8,511,7,487,7,536,7,227,8,029$ and $8,171 \mathrm{kcal} / \mathrm{kg}$ as fed.
\end{abstract}

Key Words: metabolizable energy, broilers chicks, oil, fat

\section{Introdução}

Mundialmente, temos a indústria de frangos de corte como uma das atividades que mais se destacam na receptividade de adoção de tecnologias com vistas na eficiência de produção de alimentos.

Diversos são os fatores que vêm contribuindo paulatinamente para esse contínuo estádio de desenvolvimento, que suporta ganhos cada vez menores em termos de produção, porém gigantescos em termos de produtividade. Entre esses fatores, pode-se desta- car a alta qualidade genética das linhagens, a competitividade da produção de carne com outras espécies, a eficiência alimentar das aves, que se enquadram como a única espécie consumida em todos os lugares do mundo, sem discriminação por culturas ou religiões. O consumo de carne branca tem aumentado, por ser considerada de melhor qualidade (menor teor de gorduras) que carnes vermelhas.

O uso de óleos e gorduras na nutrição de frangos de corte é amplamente discutido, tanto pelos benefícios que podem trazer, quando adicionados às dietas

\footnotetext{
${ }^{1}$ Parte da Dissertação de Mestrado da primeira autora.

2 Estudante de Doutorado da UFV, bolsista da CAPES. E.mail: cccardoso@yahoo.com

${ }^{3}$ Professores do Dep. De Zootecnia da UFV. E.mail: pcgomes@mail.uvf.br
} 
das aves, quanto pelo alto custo que esses ingredientes representam no custo total da ração. Porém, como o mercado de alimentos não obedece a nenhuma regra de constância de preços, sendo regido pelas leis da demanda e oferta, objetivou-se com este trabalho determinar os valores de energia metabolizável de alguns óleos e gorduras possíveis de serem usados pelas indústrias de ração para pintos de corte, machos e fêmeas, como alternativa de sanar problemas de ordem nutricional relacionados à eficiência produtiva de aves, visto que os benefícios podem ser obtidos a partir da adição desses alimentos à dieta das aves.

Na literatura, os valores energéticos dos alimentos variam muito. No Brasil, vários estudos já foram desenvolvidos para se determinar o valor de energia metabolizável dos alimentos utilizados em rações para aves (Lanna et al., 1979; Franqueira et al., 1979; Albino, 1980; Coelho, 1983; Albino, 1984). Rostagno et al. (2000), com base em pesquisas realizadas na Universidade Federal de Viçosa, elaboraram uma tabela de composição de alimentos, em que se observam variações nos valores de composição de alimentos quando comparados com tabelas estrangeiras. Os valores de energia metabolizável encontrados por Rostagno et al. (2000) para óleo de soja, óleo de milho, óleo de canola, gordura de aves, gordura de coco, gordura suína e sebo bovino são, respectivamente, 8790, 8886, 8826, 8817, 8330, 8570, $7374 \mathrm{kcal} / \mathrm{kg}$ de MS, enquanto os valores encontrados no NRC (1994) para óleo de soja, óleo de milho, óleo de canola, gordura de coco e sebo bovino, são, respectivamente, 8375, 9639$10811,7114-8924,8812$ e $7268-7780 \mathrm{kcal} / \mathrm{kg}$ na MS. Nesse sentido, novas pesquisas deverão ser conduzidas, visando contribuir com dados para o aprimoramento das tabelas brasileiras, uma vez que o uso da maioria dos ingredientes disponíveis no Brasil para formulação de rações de monogástricos não é o mesmo em outros países.

\section{Material e Métodos}

Foram utilizados 420 pintos de corte aos 21 dias de idade (210 machos e 210 fêmeas) da linhagem Avian Farms, alojados e separados por sexo, em baterias metálicas de $0,20 \mathrm{~m}^{2}$ contendo sete aves por unidade experimental. Essas aves, antes de serem alojadas nas baterias experimentais, foram criadas no sistema convencional de piso e receberam ração, segundo recomendações do manual da linhagem.
O delineamento experimental utilizado foi o inteiramente casualizado, em esquema fatorial $9 \times 2$, com nove alimentos a serem testados e uma dieta-referência e três repetições por sexo.

As rações testes continham os seguintes óleos e gorduras: óleo de soja degomado, óleo de milho, óleo de canola, gordura de aves, gordura de coco, gordura suína, sebo bovino, mistura de 50\% de óleo de soja degomado com $50 \%$ de gordura de coco e mistura de $50 \%$ de óleo de soja degomado com 50\% de sebo bovino. Os óleos refinados de milho e canola utilizados foram de marcas comerciais e a gordura de aves foi obtida de abatedouro avícola.

Os óleos e gorduras utilizados substituíram 9\% da ração-referência (Tabela 1). Essa ração foi formulada segundo Rostagno et al. (2000) e a composição química dos alimentos utilizados na ração foi determinada no Laboratório de Nutrição Animal do Departamento de Zootecnia da UFV.

As amostras das 10 rações foram retiradas e armazenadas para posteriores análises.

As aves receberam água e ração à vontade por dez dias, sendo os cinco primeiros de adaptação e os cinco restantes de coleta. O consumo de ração pelas aves foi devidamente registrado por unidade experimental.

O sistema de coleta total de excretas foi realizado durante cinco dias, a um intervalo de 12 horas.

Para a coleta, utilizou-se bandejas metálicas, recobertas com plástico, que foram colocadas sob cada gaiola, garantindo que não ocorresse mistura de materiais de gaiolas vizinhas, evitando-se perdas das excretas. As excretas recolhidas diariamente, durante o período experimental, foram devidamente identificadas, pesadas e armazenadas em congelador, para posteriores análises.

Ao final do período de coleta do ensaio, as excretas de cada unidade experimental foram misturadas, homogeneizadas e retiradas amostras de 400 a $500 \mathrm{~g}$ para análise.

As amostras foram levadas à estufa ventilada, durante 72 horas a uma temperatura de $60^{\circ} \mathrm{C}$, sendo posteriormente pesadas e moídas, passando por peneira de 30 mesh, para análise dos teores de matéria seca, energia bruta e nitrogênio.

Assim como foi feito com as excretas, as rações experimentais também foram amostradas, pesadas e moídas e determinados os valores de matéria seca, energia bruta e nitrogênio.

As análises das rações, fezes, óleos e gorduras 
foram realizadas no Laboratório de Nutrição Animal do Departamento de Zootecnia da UFV.

A determinação da MS e $\mathrm{N}$ foram realizadas segundo Silva (1981).

A EB foi determinada por meio de uma bomba calorimétrica.

A correção dos valores de energia metabolizável pelo balanço de nitrogênio foi feita com base nos teores de nitrogênio das amostras de rações e excretas. Foi utilizado o fator de correção de $8,22 \mathrm{kcal} / \mathrm{g}$ de $\mathrm{N}$ retido (Hill \& Anderson, 1958).

Para determinação dos valores de energia metabolizável aparente (EMA) e energia metabolizável aparente corrigida pela retenção de nitrogênio (EMAn) dos alimentos, foi utilizada a fórmula de Matterson et al. (1965).

Os dados foram analisados estatisticamente, utilizando-se o SAS (1990). As médias dos tratamentos foram comparadas pelo teste Student Newman Keuls e as médias dos sexos pelo teste $\mathrm{F}$.

\section{Resultados e Discussão}

Na Tabela 2, são apresentados os valores de matéria seca e energia bruta dos óleos e gorduras estudados. Foi observada variação nos valores de energia bruta dos óleos e gorduras estudados, conforme referenciado na literatura (Valdes \& Leeson, 1994; Fialho et al., 1995).

Os valores de energia metabolizável aparente (EMA) e energia metabolizável aparente corrigida pela retenção de nitrogênio (EMAn) dos óleos e gorduras determinados com pintos de corte machos e fêmeas encontram-se, respectivamente, nas Tabelas 3 e 4 .

Na literatura (Wiseman et al., 1986; Valdes \& Leeson, 1994), encontram-se alguns trabalhos em que são determinados os valores energéticos de óleos e gorduras. Existe certa dificuldade em se trabalhar com esses ingredientes em maiores concentrações nas rações. Além disso, o uso de óleos e gorduras nas rações animais apresenta algumas restrições por seu elevado custo, exceto quando se trata do óleo de soja degomado, cujo uso já é bastante difundido na nutrição animal. As informações sobre os valores energéticos destes alimentos são limitadas, inclusive pela inconsistência na composição dos mesmos, por falta de padronização e controle de qualidade durante as etapas de processamento e armazenamento destes produtos.
Os óleos que apresentaram melhores valores médios de EMA e EMAn $(\mathrm{P}<0,05)$ foram os óleos de canola, de milho e gordura de aves, sendo que este último não diferiu estatisticamente do óleo de soja degomado.

O óleo de soja degomado também não diferiu

Tabela 1 - Composição da ração-referência

Table 1 - Composition of the reference diet

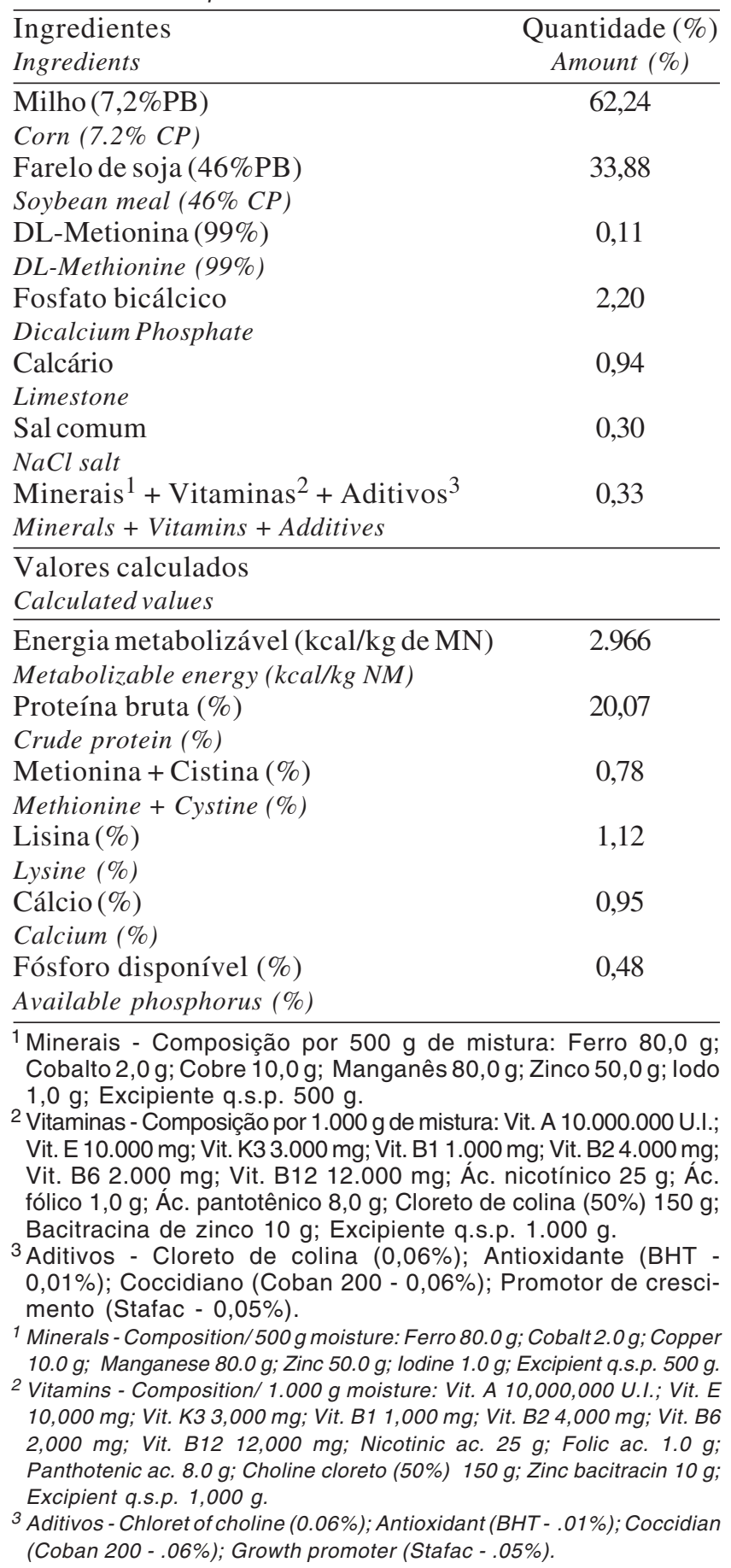


estatisticamente $(\mathrm{P}<0,05)$ das misturas de óleo de soja degomado + sebo bovino e óleo de soja degomado + gordura de coco, apesar destas misturas terem apresentado valores energéticos inferiores $(\mathrm{P}<0,05)$ aos do óleo de milho, de canola e gordura de aves. Os valores energéticos das gorduras de coco e de suíno foram estatisticamente menores $(\mathrm{P}<0,05)$ que do óleo de soja degomado e das misturas de óleo de soja degomado + sebo bovino e de óleo de soja degomado + gordura de coco, mas apresentaram valores energéticos superiores ao encontrado para o sebo bovino. Essas diferenças encontradas $(\mathrm{P}<0,05)$ entre os óleos e/ou gorduras testadas, possivelmente estejam associadas à presença de maior ou menor porcentagem de ácidos graxos, que podem ser saturados, insaturados ou poli-insaturados, presentes em diferentes proporções nos ingredientes.

Os alimentos estudados apresentaram valores de EM diferentes $(\mathrm{P}<0,01)$ para pintos de corte machos e fêmeas, sendo que um mesmo óleo ou gordura apresentou valor sempre maior para os machos, enquanto na literatura, inclusive nas tabelas de com-

Tabela 2 - Valores de matéria seca (MS) e de energia bruta (EB) dos óleos e gorduras ${ }^{1}$

Table 2 - Dry matter (DM) and gross energy (GE) values of oils and fats

\begin{tabular}{|c|c|c|}
\hline $\begin{array}{l}\text { Óleos e Gorduras } \\
\text { Oils and Fats }\end{array}$ & $\begin{array}{l}\mathrm{MS}(\%) \\
D M(\%)\end{array}$ & $\begin{array}{c}\mathrm{EB}(\mathrm{kcal} / \mathrm{kg})^{2} \\
C E(k c a l / k g)\end{array}$ \\
\hline $\begin{array}{l}\mathrm{OSD}^{3} \\
\mathrm{DSO}\end{array}$ & 99,3 & 9225,12 \\
\hline Óleo de milho & 99,1 & 9247,41 \\
\hline $\begin{array}{l}\text { Óleo de canola } \\
\text { Canola oil }\end{array}$ & 99,3 & 9359,33 \\
\hline $\begin{array}{l}\text { Gordura de aves } \\
\text { Avian fat }\end{array}$ & 99,6 & 9230,14 \\
\hline $\begin{array}{l}\text { Gordura de coco } \\
\text { Coconutfat }\end{array}$ & 99,1 & 8977,13 \\
\hline $\begin{array}{l}\text { Gordura de suínos } \\
\text { Swine fat }\end{array}$ & 99,6 & 9322,07 \\
\hline $\begin{array}{l}\text { Sebo bovino } \\
\text { Beeftallow }\end{array}$ & 99,3 & 9390,72 \\
\hline $\begin{array}{l}\text { OSD + gordura de coco }(1: 1) \\
D S O+\text { coconut fat }(1: 1)\end{array}$ & 99,4 & 9142,73 \\
\hline $\begin{array}{l}\text { ÓSD + sebo bovino }(1: 1) \\
\text { DSO + beef tallow }(1: 1)\end{array}$ & 99,5 & 9367,58 \\
\hline
\end{tabular}

${ }^{1}$ Análises realizadas no Laboratório de Nutrição Animal (Departamento de Zootecnia da UFV).

2 Valores expressos na matéria natural.

3 Óleo de soja Degomado.

${ }^{1}$ Analyses were performed at the Animal Nutrition Laboratory (Animal Science Department/UFV).

2 Values expressed in the natural matter.

${ }^{3}$ Degumed soybean oil. posição dos alimentos (NRC,1994; Rostagno et al., 2000 e EMBRAPA, 1991), não é feita diferenciação para sexo, sendo os valores calculados para lotes mistos ou somente para machos. Esse resultado vem apoiar a necessidade e eficiência de criação de frangos com sexos separados após 14 dias de idade, conforme descrito por Larbier \& Lerclercq (1994), citados por Tardin (1995).

Os valores médios de EMA e EMAn do óleo de soja degomado de $8.336 \pm 205$ e $8.331 \pm 204 \mathrm{kcal} / \mathrm{kg}$, respectivamente, foram semelhantes àqueles citados pelo NRC (1994) e por Albino et al. (1993), superiores aos citados por Albino et al. (1986a, b) e Zanotto et al. (1995b) e inferiores aos citados por Rostagno et al. (2000), Valdes \& Leeson (1994) e Blanch et al. (1995).

Para o óleo de milho, os valores médios de EMA e EMAn obtidos foram de $8.666 \pm 629$ e $8660 \pm 508 \mathrm{kcal} / \mathrm{kg}$. Esses valores foram semelhantes aos citados por Rostagno et al. (1983) e inferiores àqueles citados pelo NRC (1994) e Valdes \& Leeson (1994).

O óleo de canola apresentou valores médios de EMA iguais a $8.825 \pm 550 \mathrm{kcal} / \mathrm{kg}$ e de EMAn igual a $8.742 \pm 630 \mathrm{kcal} / \mathrm{kg}$, valores abaixo do recomendado pelo NRC (1994) e acima dos observados por Valdes \& Leeson (1994).

A gordura de coco teve seus valores médios de EMA e EMAn correspondentes a 7.522 \pm 141 e 7.517 \pm 139 , respectivamente. Esses valores foram inferiores aos reportados no NRC (1994), porém próximos dos citados por Valdes \& Leeson (1994). A diferença encontrada talvez possa ser explicada com base na matéria-prima utilizada, seja pela fonte da mesma, pela técnica de processamento ou ainda pela época em que as análises foram realizadas.

Entre os alimentos de origem animal, a gordura suína e o sebo bovino apresentaram valores médios de EMA e EMAn, respectivamente, iguais a $7.594 \pm$ 360 e $7.589 \pm 358 \mathrm{kcal} / \mathrm{kg}$ para a gordura suína e 7.282 \pm 105 e $7.278 \pm 103 \mathrm{kcal} / \mathrm{kg}$ para o sebo bovino, em conformidade com os valores citados por Albino (1986), NRC (1994) e Valdes \& Leeson (1994). No caso do sebo bovino, o valor médio encontrado foi menor que o estimado pelo NRC (1994). Para gordura de aves, os valores médios de EMA $(8.553 \pm 290 \mathrm{kcal} /$ $\mathrm{kg})$ e EMAn $(8.545 \pm 290 \mathrm{kcal} / \mathrm{kg})$ foram inferiores aos descritos por Valdes \& Leeson (1994).

Quando se estudou o efeito da mistura de dois tipos de óleos ou gorduras sobre o valor energético dos mesmos, observou-se melhoria no teor de energia, que pode ser explicada pelo efeito sinérgico ou

\section{R. Bras. Zootec., v.33, n.2, p.375-381, 2004}


dos ácidos graxos poli-insaturados da dieta, auxiliando na absorção dos ácidos saturados ou de cadeias longas (Leeson \& Summers, 1976; Freeman, 1984; Wiseman et al., 1986). As misturas de óleo de soja degomado + gordura de coco e óleo de soja degomado + sebo bovino, ambas na proporção de 1:1, apresentaram valores médios de EMA e EMAn, respectivamente, para a primeira mistura, de $8.082 \pm 218$ e $8.077 \pm 218 \mathrm{kcal} / \mathrm{kg}$ e, para a segunda mistura, de $8.216 \pm 367$ e $8.212 \pm 366 \mathrm{kcal} / \mathrm{kg}$. Esses valores encontrados estão em concordância com a premissa acima citada, já que a adição de óleo de soja à gordura de coco e ao sebo bovino melhora a digestibilidade de ambos ingredientes. Observa-se, na composição de ácidos graxos dos ingredientes acima, alta porcentagem de ácidos graxos saturados (Sell et al., 1986; Brown et al., 1993; NRC, 1994; Ding e Lilburn, 1997), o que ser explicado pelo fato de os ácidos graxos insaturados, em relação aos saturados, serem mais absorvidos no intestino delgado. Esse efeito dos ácidos graxos insaturados e poli-insaturados melhorando a digestibilidade dos ácidos graxos saturados também é relatado nos trabalhos de Scott et al. (1976), Guirguis (1976), Mateos \& Sell (1980ab), Morita (1992), Young \& Garret (1963), Artman (1964) e Wiseman \& Lessire (1987), citados por Amaral Neto (1999).

Além do aspecto nutricional, a mistura de óleo de soja degomado com sebo bovino deve ser avaliada como forma de redução nos custos da ração. Se os óleos de origem vegetal são de custo mais elevado que as gorduras de origem animal e o valor energético da mistura de óleo de soja degomado + sebo bovino na proporção de 1:1 é estatisticamente igual $(\mathrm{P}<0,05)$ ao valor energético do óleo de soja degomado, essa pode ser uma alternativa econômica de grande importância para a indústria que precisa reduzir seus custos de produção.

Tabela 3 - Valores de energia metabolizável aparente (EMA) dos óleos e gorduras, com os respectivos desvios-padrão, para pintos de corte machos e fêmeas ${ }^{1}$

Table 3 - Apparent metabolizable energy values (AME) of oils and fats, with the respective standard errors (SE), for broiler chicks males and females

\begin{tabular}{|c|c|c|c|}
\hline \multirow[b]{2}{*}{$\begin{array}{l}\text { Alimentos } \\
\text { Feedstuffs } \\
\end{array}$} & \multicolumn{2}{|c|}{$\begin{array}{l}\text { Pintos de corte } \\
\text { Broilers chickens }\end{array}$} & \multirow[b]{2}{*}{$\begin{array}{l}\text { Média } \\
\text { Means }\end{array}$} \\
\hline & $\begin{array}{l}\text { Machos } \\
\text { Males }\end{array}$ & $\begin{array}{l}\text { Fêmeas } \\
\text { Females }\end{array}$ & \\
\hline $\begin{array}{l}\mathrm{OSD}^{2} \\
\mathrm{DSO}^{2}\end{array}$ & $8.410 \pm 130$ & $8.262 \pm 280$ & $8.336 \pm 205 b c$ \\
\hline $\begin{array}{l}\text { Óleo de milho } \\
\text { Corn oil }\end{array}$ & $8.762 \pm 413$ & $8.570 \pm 845$ & $8.666 \pm 629 a$ \\
\hline $\begin{array}{l}\text { Óleo de canola } \\
\text { Canola oil }\end{array}$ & $8.895 \pm 723$ & $8.754 \pm 378$ & $8.825 \pm 550 \mathrm{a}$ \\
\hline $\begin{array}{l}\text { Gordura de aves } \\
\text { Avian fat }\end{array}$ & $8.587 \pm 283$ & $8.520 \pm 297$ & $8.553 \pm 290 \mathrm{ab}$ \\
\hline $\begin{array}{l}\text { Gordura de coco } \\
\text { Coconutfat }\end{array}$ & $7.668 \pm 36$ & $7.376 \pm 246$ & $7.522 \pm 141 \mathrm{~d}$ \\
\hline $\begin{array}{l}\text { Gordura de suínos } \\
\text { Swine fat }\end{array}$ & $7.676 \pm 295$ & $7.512 \pm 425$ & $7.594 \pm 360 d$ \\
\hline $\begin{array}{l}\text { Sebo bovino } \\
\text { Beeftallow }\end{array}$ & $7.311 \pm 107$ & $7.253 \pm 104$ & $7.282 \pm 105 \mathrm{e}$ \\
\hline $\begin{array}{l}\text { OSD + gordura de coco }(1: 1) \\
D S O+\text { coconut fat }(1: 1)\end{array}$ & $8.146 \pm 319$ & $8.019 \pm 117$ & $8.082 \pm 218 c$ \\
\hline $\begin{array}{l}\text { ÓSD + sebo bovino }(1: 1) \\
\text { DSO + beef tallow }(1: 1)\end{array}$ & $8.228 \pm 618$ & $8.202 \pm 116$ & $8.216 \pm 367 \mathrm{c}$ \\
\hline $\begin{array}{l}\text { Média } \\
\text { Mean }\end{array}$ & $8187 \pm 545 \mathrm{~A}$ & $8035 \pm 530 \mathrm{~B}$ & $\mathrm{CV}=2,35$ \\
\hline
\end{tabular}

\footnotetext{
1 Valores expressos em $\mathrm{kcal} / \mathrm{kg}$ de matéria natural.

2 Óleo de soja degomado.

Médias seguidas pela mesma letra minúscula não diferem $(P<0,05)$ em uma mesma coluna - Teste Student Newman Keuls.

Médias seguidas pela mesma letra maiúscula não diferem $(P<0,01)$ em uma mesma linha - Teste $F$.

1 Values expressed in $\mathrm{kcal} / \mathrm{kg}$ of natural matter.

${ }^{2}$ Degumed soybean oil.

Means followed by the same small letter do not differ $(P<.05)$ in the same column - Student Newman Keuls test.

Means followed by the same capital letter do not differ $(P<.01)$ in the same row - $F$ test.
}

R. Bras. Zootec., v.33, n.2, p.375-381, 2004 
Tabela 4 - Valores de energia metabolizável aparente corrigida para a retenção de nitrogênio (EMAn) dos óleos e gorduras, com os respectivos desvios-padrão, para pintos de corte machos e fêmeas ${ }^{1}$

Table 4 - Values of nitrogen-corrected apparent metabolizable energy (AMEn) of the oils and fats, with the respective standard errors (SE), for male and female broiler chicks

\begin{tabular}{|c|c|c|c|}
\hline \multirow{3}{*}{$\begin{array}{l}\text { Alimentos } \\
\text { Feedstuffs }\end{array}$} & \multicolumn{3}{|c|}{$\begin{array}{l}\text { Pintos de corte } \\
\text { Broiler chicks }\end{array}$} \\
\hline & Machos & Fêmeas & Média \\
\hline & Males & Females & Mean \\
\hline OSD2 & $8.403 \pm 132$ & $8.258 \pm 276$ & $8.331 \pm 204 b c$ \\
\hline $\mathrm{DSO} 2$ & & & \\
\hline Óleo de milho & $8.755 \pm 412$ & $8.565 \pm 605$ & $8.660 \pm 508 \mathrm{a}$ \\
\hline Corn oil & & & \\
\hline Óleo de canola & $8.889 \pm 718$ & $8.595 \pm 542$ & $8.742 \pm 630 a$ \\
\hline Canola oil & & & \\
\hline Gordura de aves & $8.578 \pm 284$ & $8.512 \pm 296$ & $8.545 \pm 290 \mathrm{ab}$ \\
\hline Avian fat & & & \\
\hline Gordura de coco & $7.660 \pm 34$ & $7.373 \pm 245$ & $7.517 \pm 139 \mathrm{~d}$ \\
\hline $\begin{array}{l}\text { Coconutfat } \\
\text { Gordura de suínos }\end{array}$ & $7.668 \pm 294$ & $7.509 \pm 422$ & $7.589 \pm 358 \mathrm{~d}$ \\
\hline Swine fat & & & \\
\hline $\begin{array}{l}\text { Sebo bovino } \\
\text { Beeftallow }\end{array}$ & $7.304 \pm 106$ & $7.252 \pm 101$ & $7.278 \pm 103 \mathrm{e}$ \\
\hline $\begin{array}{l}\text { OSD + gordura de coco }(1: 1) \\
D S O+\text { coconut fat }(1: 1)\end{array}$ & $8.138 \pm 321$ & $8.016 \pm 116$ & $8.077 \pm 218 c$ \\
\hline $\begin{array}{l}\text { ÓSD + sebo bovino }(1: 1) \\
D S O+\text { beef tallow }(1: 1)\end{array}$ & $8.221 \pm 616$ & $8.202 \pm 116$ & $8.212 \pm 366 c$ \\
\hline $\begin{array}{l}\text { Média } \\
\text { Mean }\end{array}$ & $8180 \pm 54 \mathrm{~A}$ & $8031 \pm 529 \mathrm{~B}$ & $\mathrm{CV}=2,34$ \\
\hline
\end{tabular}

${ }^{1}$ Valores expressos em $\mathrm{kcal} / \mathrm{kg}$ de matéria natural.

2 Óleo de soja degomado.

Médias seguidas pela mesma letra minúscula não diferem $(P<0,05)$ em uma mesma coluna - Teste Student Newman Keuls.

Médias seguidas pela mesma letra maiúscula não diferem $(P<0,01)$ em uma mesma linha - Teste $F$.

1 Values expressed in $\mathrm{kcal} / \mathrm{kg}$ of natural matter.

2 Degumed soybean oil.

Means followed by the same small letter do not differ $(P<.05)$ in the same column - Student Newman Keuls test.

Means followed by the same capital letter do not differ $(P<.01)$ in the same row - $F$ test.

\section{Conclusões}

Os valores de EMA e EMAn foram estatisticamente maiores $(\mathrm{P}<0,01)$ para os machos em relação às fêmeas, sendo os valores encontrados para as fêmeas 98\% dos encontrados para os machos.

Os valores de EMA e EMAn na MN das misturas de óleo de soja degomado + gordura de coco e óleo de soja degomado + sebo bovino, ambas na proporção de 1:1, mostram o sinergismo existente entre ácidos graxos insaturados e saturados. Houve aumento de $560 \mathrm{kcal} / \mathrm{kg}$ na mistura de óleo de soja à gordura de coco e de $934 \mathrm{kcal} / \mathrm{kg}$, quando esta foi adicionada ao sebo bovino.

Os valores médios de EMA e EMAn de óleo de soja degomado, óleo de milho, óleo de canola, gordura de aves, gordura de coco, gordura de suíno, sebo bovino, óleo de soja degomado + gordura de coco e óleo de soja degomado + sebo bovino, para pintos de corte, são, respectivamente: 8.336 e $8.331 ; 8.666$ e $8660 ; 8.825$ e $8.742 ; 8.553$ e $8.545 ; 7.522$ e 7.517 ; 7.594 e $7.589 ; 7.282$ e $7.278 ; 8.082$ e 8.077 ; e 8.216 e $8.212 \mathrm{kcal} / \mathrm{kg}$ na $\mathrm{MN}$.

\section{Literatura Citada}

ALBINO, L.F.T. Determinação de valores de energia metabolizável e triptofano de alguns alimentos para aves em diferentes idades. Viçosa, MG: Universidade Federal de Viçosa, 1980. 55p. Dissertação (Mestrado em Zootecnia) - Universidade Federal de Viçosa, 1980.

ALBINO, L.F.T.; FIALHO, E.T.; ROSA, P.S. Composição química e valores energéticos alguns alimentos para frangos de corte. Pesquisa Agropecuária Brasileira, v.19, n.7, p.897 -903, 1984.

ALBINO, L.F.T.; FIALHO, E.T.; BLUME, E. Energia metabolizável e composição química e valores energéticos alguns alimentos para frangos de corte. Revista da Sociedade Brasileira de Zootecnia, v.15, n.3, p.185-193, 1986a.

ALBINO, L.F.T.; COELHO, M.G.R.; RUTZ, F.; BRUM, P.A.R. Determinação da energia metabolizável de alimentos com

\section{R. Bras. Zootec., v.33, n.2, p.375-381, 2004}


frangos de corte. In: REUNIÃO ANUAL DA SOCIEDADE BRASILEIRA DE ZOOTECNIA, 23., 1986, Campo Grande. Anais... Campo Grande: Sociedade Brasileira de Zootecnia, 1986b. p.68.

ALBINO, L.F.T.; BRUM, P.A.R.; GOMES, P.C. Avaliação energética de alguns alimentos para aves. In: REUNIÃO ANUAL DA SOCIEDADE BRASILEIRA DE ZOOTECNIA, 30., 1993, Rio de Janeiro. Anais... Rio de Janeiro: Sociedade Brasileira de Zootecnia, 1993. p. 312.

AMARAL NETO, E.B. Antioxidantes na conservação das características nutricionais de alimentos usados em rações para aves. Viçosa, MG: Universidade Federal de Viçosa, 1999. 66p. Tese (Doutorado em Zootecnia) - Universidade Federal de Viçosa, 1999.

BLANCH, A.; BARROETA, A.C.; BAUCELLS, M.D. et al. The nutritive value of dietary fats in relation to their chemical composition. Apparent fat availability and metabolizable energy in two-week-old chicks. Poultry Science, v.74, p.1335-1340, 1995.

BROWN, P.K.; POTTER, L.M.; WATKINS, B.A. Metabolizable energy values of soybean oil and hydrogenated soybean oil for broilers. Poultry Science, v.72, p.794-797, 1993.

COELHO, M.G.R. Valores energéticos e de triptofano metabolizável de alimentos para aves, utilizando duas metodologias. Viçosa, MG: Universidade Federal de Viçosa, 1983. 77p. Dissertação (Mestrado em Zootecnia) Universidade Federal de Viçosa, 1983.

DING, S.T.; LILBURN, M.S. Inclusion of coconut oil in diets for turkeys breeders and its effects on embryonic yolk and liver fatty acids. Poultry Science, v.76, p.1714-1721, 1997.

EMPRESA BRASILEIRA DE PESAQUISA AGROPECUÁRIA - EMBRAPA - CNPSA. Tabela de composição química e valores energéticos de alimentos para suínos e aves. 3 . ed. Concórdia, 1991. p.97.

FIALHO, E.T.; LIMA, J.A.F.; FERREIRA, R.A. et al. Determinação dos valores energéticos de óleos e gorduras de origem animal para suínos através de ensaios metabólicos. In: REUNIÃO ANUAL DA SOCIEDADE BRASILEIRA DE ZOOTECNIA, 32., 1995, Brasília. Anais... Brasília: Sociedade Brasileira de Zootecnia, 1995. p.520.

FRANQUEIRA, J.M.; ROSTAGNO, H.S.; SILVA, D.J. et al. Tabela brasileira de composição de alimentos concentrados. IV. Rações de mínimo custo para poedeiras calculadas com diferentes valores de composição química e de energia metabolizável. Revista da Sociedade Brasileira de Zootecnia, v.8, n.4, p.697-708, 1979.

FREEMAN, C.P. The digestion, absorption and transport of fat - Non - ruminants. In: WISEMAN, J. (Ed.) Fats in animal nutrition. London: Butterworths, 1984. p.105-122.

GUIRGUIS, N. Metabolizable energy values of fat and protein concentrates for poultry; effect of Sex and inclusion level of feedstuffs. Australian Journal Experimental Agricultural and Animal Husbandry, v.16, p.691-695, 1976.

HILL, F.W.; ANDERSON, D.L. Comparison of the metabolizable energy nd productive energy determinations for the chicks. Journal Nutrition, v.64, p.587-603, 1958.
LANNA, P.A.S.; ROSTAGNO, H.S.; SILVA, D.J. et al. Tabela de composição de alimentos concentrados. I. Valores de composição química e de energia metabolizável determinados com pintos. Revista da Sociedade Brasileira de Zootecnia, v.8, n.3, p.516-523, 1979.

LEESON, S.; SUMMERS, J.D. Fat ME values: the effect of fat acid saturation. Feedstuffs, v.48, p.26-28, 1976.

MATTEOS, G.G.; SELL, J.L. Influence of carbohidrate and supplemental fat source on the metabolizable energy of the diet. Poultry Science, v.59, n.9, p.2129-2135, 1980a.

MATTEOS, G.G.; SELL, J.L. True the apparent metabolizable energy value of fat for laying hens. Influence of level of use. Poultry Science, v.59, n.2, p.369-373, 1980b.

MATTERSON, L.D.; POTTER, L.M.; STUTZ, M.W. et al. The metabolizable energy of feed ingredients for chickens. University of Connecticut Storrs. Agricultural Experimental Station Research Report, v.7, p.11, 1965.

MORITA, M..M. Custo x benefício do uso de óleos e gorduras em rações avícolas. In: CONFERÊNCIA APINCO DE CIÊNCIA E TECNOLOGIA AVÍCOLA, 1992, Santos. Anais... Santos: FACTA, 1992. p.29-35.

NATIONAL RESEARCH COUNCIL - NRC. Nutrient requeriments of poultry. 9. ed. Washington, D. C.: National Academic Press, 1994. 155p.

ROSTAGNO, H.S.; SILVA, D.J.; COSTA, P.M.A. et al. Composição de alimentos e exigências nutricionais de aves e suínos, tabelas brasileiras. Viçosa, MG: Universidade Federal de Viçosa, 1983. 61p.

STATISTICAL ANALYSES SYSTEM - SAS. User's guide: statistics. version 6. 4.ed. Cary: 1990. 1686p.

SCOTT, M.L.; NESHEIM, M.C.; YOUNG, R.J. Nutrition of the chicken. Ithaca, N. Y.: 1976. 511 p.

SILVA, D.J. Análise de alimentos: métodos químicos e biológicos. Viçosa, MG: Universidade Federal de Viçosa, 1981. $165 \mathrm{p}$.

TARDIN, A.C. Conceituação e importância da energia na nutrição das aves. In: SIMPÓSIO INTERNACIONAL SOBRE AMBIÊNCIA E INSTALAÇÃO NA AVICULTURA INDUSTRIAL, 1995, Campinas. Anais... Campinas: 1995. p.213-239.

VALDES, E.V.; LEESON, S. Measurement of metabolizable energy, gross energy, and moisture in feed grade fats by near infrared reflectance spectroscopy. Poultry Science, v.73, p. 163-171, 1994.

WISEMAN, J.; COLE, D.J.A.; PERRY, F.G. et al. Apparent metabolizable energy values of fats for broiler chicks. Poultry Science, v.27, p.561-576, 1986.

ZANOTTO, D.L.; BRUM, P.A.R.; LIMA, G.J.M.M. Determinação de valores de composição química e de energia metabolizável de alimentos para aves. In: CONFERÊNCIA APINCO DE CIÊNCIA E TECNOLOGIA AVÍCOLA, 1995, Campinas. Anais... Campinas: FACTA, 1995. p.65-66.

Recebido em: 13/11/02 Aceito em: 09/07/03 